\title{
Desconfortos Relatados Pelos Pacientes Após Cateterismo Cardíaco Pelas Vias Femoral ou Radial
}

\author{
Caroline Dal Piva', Eliane Vaz¹, Maria Antonieta Moraes¹, Silvia Goldmeyer ${ }^{1}$, \\ Graciele Fernanda da Costa Linch², Emiliane Nogueira de Souza ${ }^{2}$
}

\section{RESUMO}

Introdução: Queixas são frequentes por parte dos pacientes submetidos a procedimentos invasivos percutâneos. Nosso objetivo foi verificar os desconfortos de pacientes submetidos a cateterismo cardíaco pelas vias femoral e radial. Métodos: Estudo transversal, com amostra não probabilística de adultos submetidos a cateterismo. Os dados foram coletados por meio de questionário. Resultados: Foram estudados 228 pacientes, sendo 205 que realizaram procedimento pela via femoral e 23 pela radial. Em todos os pacientes, foi utilizado o introdutor arterial 6 F. A média de idades foi de $60,0 \pm 11,5$ anos, e a maioria era do sexo masculino $(50,4 \%)$. As principais queixas foram dor lombar $(65,8 \%)$ e mal-estar $(32,0 \%)$. Em uma escala de zero a 10, o valor médio atribuído para a intensidade de dor lombar foi de 5,0 $\pm 4,2$ e de 1,5 $\pm 2,7$ para dor no local da punção. Pacientes que realizaram o procedimento pela via radial referiram ter mais dor no local da punção do que os que utilizaram a via femoral $(26,8 \%$ vs. $52,2 \% ; p=0,01)$. No entanto, os pacientes abordados por via femoral relataram mais frequentemente dor lombar $(69,8 \%$ vs. $30,4 \% ; p<0,01)$ e mal-estar $(34,6 \%$ vs. $8,7 \% ; p=0,01)$. Conclusões: $\mathrm{O}$ desconforto predominante pós-punção femoral foi a lombalgia e, naqueles submetidos à punção radial, foi a dor no local da punção. Nossos achados remetem a recomendações para a prática clínica que promova a qualidade da assistência, como instituir medidas de conforto ao paciente, como o uso de coxins, mudança de decúbito, auxílio na deambulação e criação de um ambiente acolhedor.

DESCRITORES: Cateterismo cardíaco. Artéria radial. Artéria femoral. Dor. Cuidados de enfermagem.

\section{ABSTRACT}

Discomfort Reported by Patients After Cardiac Catheterization Using the Femoral or Radial Approaches

Background: Complaints of patients undergoing invasive percutaneous procedures are a frequent finding. Our objective was to assess the discomfort of patients undergoing cardiac catheterization using femoral or radial approach. Methods: Cross-sectional study with a non-probabilistic sample of adults undergoing catheterization. Data were collected through a questionnaire. Results: We included 228 patients, 205 who underwent the procedure via the femoral approach and 23 by the radial approach. A $6 \mathrm{~F}$ arterial sheath was used in all patients. Mean age was $60.0 \pm 11.5$ years and most of them were male $(50.4 \%)$. The main complaints were lumbar pain in $65.8 \%$ and malaise in $32.0 \%$ of the cases. In a scale of 0 to 10 , the average value assigned for lumbar pain intensity was $5.0 \pm 4.2$ and $1.5 \pm 2.7$ for pain at the puncture site. Patients who used the radial approach reported having more pain at the puncture site than patients who used the femoral access $(26.8 \%$ vs. $52.2 \% ; p=0.01)$. However, patients who used the femoral approach most often reported lumbar pain $(69.8 \%$ vs. $30.4 \% ; p<0.01)$ and malaise $(34.6 \%$ vs. $8.7 \%$; $p=0.01)$. Conclusions: The predominant discomfort after femoral puncture was lumbar pain and in patients undergoing radial puncture it was pain at the access site. Our findings corroborate the recommendations for a clinical practice that promotes better patient care, including comfort measures, such as the use of cushions, changes in body position, supervised ambulation and the creation of a welcoming environment.

DESCRIPTORS: Cardiac catheterization. Radial artery. Femoral artery. Pain. Nursing care.

\footnotetext{
1 Instituto de Cardiologia do Rio Grande do Sul/Fundação Universitária de Cardiologia, Porto Alegre, RS, Brasil.

2 Universidade Federal de Ciências da Saúde de Porto Alegre, Porto Alegre, RS, Brasil.
}

Correspondência: Emiliane Nogueira de Souza. Avenida Princesa Isabel, 370 - Santana - CEP: 90620-000 - Porto Alegre (RS), Brasil

E-mail: enogsouza@hotmail.com

Recebido em: 16/2/2013 • Aceito em: 1/2/2014 
E tudos têm demonstrado que, apesar do crescente avanço tecnológico, e da utilização de técnicas contemporâneas de intervenção coronária, diagnóstica ou terapêutica, ainda são observados desconfortos relacionados aos procedimentos. ${ }^{1,2} \mathrm{~A}$ via radial é geralmente a opção preferida pelos pacientes, em virtude do maior conforto que traz, comparada ao procedimento realizado por via femoral. ${ }^{3}$ No entanto, o acesso femoral ainda é a via de escolha do operador, propiciando procedimentos mais rápidos, permitindo intervenções repetidas e o uso de uma maior variabilidade de materiais, e exigindo menor treinamento em relação ao acesso radial. ${ }^{1}$ Porém, a opção por essa via demanda um período de restrição ao leito, que leva a desconfortos adicionais, além de a permanência hospitalar ser mais longa. ${ }^{4} \mathrm{O}$ acesso radial, por outro lado, tem como vantagem a deambulação precoce, mas apresenta, entre suas limitações, a dificuldade da punção e o pequeno calibre da artéria. $^{5}$

Observa-se que as manifestações dos pacientes em relação à permanência prolongada no leito em decúbito dorsal acarretam dores lombares e dificuldade para eliminações fisiológicas, além de dor durante a compressão da via de acesso. A busca da natureza dessas queixas, sob a ótica do paciente, justifica a realização deste estudo. Assim, nosso objetivo foi descrever os desconfortos dos pacientes submetidos a cateterismo cardíaco, comparando as vias de acesso femoral e radial.

\section{MÉTODOS}

Estudo com delineamento transversal, realizado com pacientes submetidos a cateterismo cardíaco pelas vias de acesso radial ou femoral, em um serviço privado de Hemodinâmica do Rio Grande do Sul, nos meses de abril a junho de 2009. Esse serviço conta com uma enfermeira no turno da manhã e tarde e seis técnicos de enfermagem divididos nos dois turnos. A amostra foi do tipo não probabilística, tendo sido incluídos todos os pacientes que aceitaram participar do estudo, de ambos os sexos, com idade $\geq 18$ anos e que não apresentassem complicações durante o procedimento.

Os dados foram coletados antes de os pacientes receberem alta da Hemodinâmica para casa ou para sua unidade de origem (terapia intensiva ou internação clínica). No momento da coleta de dados, a qual foi realizada por uma das enfermeiras pesquisadoras, explicou-se a relevância do estudo, solicitando a participação dos pacientes. O questionário foi aplicado após o término do repouso para os pacientes submetidos a procedimentos pela via de acesso femoral, que era de 6 horas, e de 3 horas para os pacientes submetidos a procedimentos pela via de acesso radial.

As variáveis independentes pesquisadas foram: dados sociodemográficos, variáveis clínicas e relacionadas ao procedimento. As variáveis dependentes foram as seguintes: dor lombar, dificuldade urinária, dificuldade para deambular, constrangimento, hematoma, equimose, mal-estar, sangramento, náusea e vômito. A dor no local da punção foi avaliada como quinto sinal vital, observando a intensidade por meio da escala linear de dor para posterior comparação entre os grupos (zero para ausência total de dor; 1 a 3 para dor de fraca intensidade; 4 a 6 para intensidade moderada; 7 a 9 para forte intensidade; e 10 para dor de intensidade insuportável). ${ }^{2}$ As complicações vasculares, tais como hematoma, equimose e sangramento leve, foram consideradas de acordo com a literatura. ${ }^{6}$ Dificuldade urinária foi avaliada como défice ou incapacidade de urinar durante repouso.

Os dados foram analisados pelo programa estatístico Statistical Package for the Social Science (SPSS), versão 14.0. As variáveis contínuas foram descritas como média \pm desvio padrão. As variáveis categóricas foram descritas como frequências absolutas (n) e relativas (\%). Para comparar médias entre variáveis com distribuição normal, foi utilizado o teste $t$, e para comparar variáveis categóricas, foi utilizado o teste qui-quadrado. Considerou-se como de significância estatística um $p<0,05$.

Este estudo foi aprovado pelo Comitê de Ética e Pesquisa do Instituto de Cardiologia do Rio Grande do Sul, sob o número 4246/08. Todos os pacientes foram incluídos após assinarem o Termo de Consentimento Livre e Esclarecido.

\section{RESULTADOS}

Dos 232 pacientes considerados para o protocolo, 4 foram excluídos da análise devido à ocorrência de acidente vascular cerebral isquêmico, bradicardia, hipotensão e reação alérgica. Dessa maneira, 228 pacientes foram avaliados, sendo que, destes, 205 realizaram procedimento pela via de acesso femoral e 23 pela via de acesso radial. Em todos os pacientes, foi utilizado introdutor arterial $6 \mathrm{~F}$. A média de idade dos pacientes foi de $60,0 \pm 11,5$ anos, sendo a maioria $(50,4 \%)$ do sexo masculino. Demais características avaliadas podem ser visualizadas na tabela 1 .

Do total de pacientes da amostra, $70(30,7 \%)$ já tinham realizado cateterismo cardíaco, 33(14,5\%) intervenção coronária percutânea e 11 (4,8\%) cirurgia de revascularização do miocárdio prévios.

Em uma escala de zero a 10, o valor médio atribuído pelos pacientes para a intensidade de dor lombar foi de 5,0 \pm 4,2 e de 1,5 $\pm 2,7$ para dor no local da punção. Os pacientes que realizaram o procedimento pela via radial referiram ter mais dor no local da punção do que aqueles que utilizaram a via femoral $(26,8 \%$ vs. $52,2 \% ; p=0,01)$. No entanto, os pacientes abordados por via femoral relataram dor lombar mais frequentemente $(69,8 \%$ vs. $30,4 \% ; p<0,01)$ e mal-estar geral $(34,6 \%$ vs. $8,7 \% ; p=0,01)$. Estes dados estão demonstrados na Tabela 2 . 
TABELA 1

Características sociodemográficas e clínicas

\begin{tabular}{|c|c|c|c|c|}
\hline Variáveis & $\begin{array}{c}\text { Total } \\
(n=228)\end{array}$ & $\begin{array}{c}\text { Femoral } \\
(n=205)\end{array}$ & $\begin{array}{c}\text { Radial } \\
(n=23)\end{array}$ & Valor de $p$ \\
\hline Sexo masculino, $\mathrm{n}(\%)$ & $115(50,4)$ & $103(50,2)$ & $12(52,2)$ & 0,86 \\
\hline Hipertensão arterial, n (\%) & $171(75,0)$ & $153(74,6)$ & $18(78,3)$ & 0,70 \\
\hline Dislipidemia, n (\%) & $80(35,1)$ & $70(34,1)$ & $10(43,5)$ & 0,37 \\
\hline Infarto do miocárdio prévio, n (\%) & $28(12,3)$ & $25(12,2)$ & $3(13,0)$ & 0,90 \\
\hline Insuficiência cardíaca, n (\%) & $7(3,1)$ & $7(3,4)$ & 0 & 0,36 \\
\hline Doença pulmonar obstrutiva crônica, n (\%) & $6(2,6)$ & $5(1,8)$ & $1(3,4)$ & 0,44 \\
\hline
\end{tabular}

TABELA 2

Desconfortos e complicações relatados pelos pacientes

\begin{tabular}{|c|c|c|c|c|}
\hline Desconfortos & $\begin{array}{c}\text { Total } \\
(\mathrm{n}=\mathbf{2 2 8})\end{array}$ & $\begin{array}{c}\text { Femoral } \\
(n=205)\end{array}$ & $\begin{array}{c}\text { Radial } \\
(n=23)\end{array}$ & Valor de $p$ \\
\hline Dor lombar, n (\%) & $150(65,8)$ & $143(69,8)$ & $7(30,4)$ & $<0,01$ \\
\hline Mal-estar geral, n (\%) & $73(32,0)$ & $71(34,6)$ & $2(8,7)$ & 0,01 \\
\hline Dor no local da punção, n (\%) & $67(29,4)$ & $55(26,8)$ & $12(52,2)$ & 0,01 \\
\hline Dificuldade para urinar, $\mathrm{n}(\%)$ & $64(28,1)$ & $60(29,3)$ & $4(17,4)$ & 0,52 \\
\hline Constrangimento, n (\%) & $35(15,4)$ & $32(16,1)$ & $2(8,7)$ & 0,35 \\
\hline Náusea, n (\%) & $23(10,1)$ & $21(10,2)$ & $2(8,7)$ & 0,81 \\
\hline Dificuldade para deambular, n (\%) & $11(4,8)$ & $10(4,9)$ & $1(4,3)$ & 0,83 \\
\hline Hematoma, n (\%) & $6(3,0)$ & $4(2,0)$ & $2(8,7)$ & 0,06 \\
\hline Vômito, n (\%) & $7(3,1)$ & $7(3,4)$ & 0 & 0,36 \\
\hline Equimose, $\mathrm{n}(\%)$ & $4(2,0)$ & $3(1,5)$ & $1(4,3)$ & 0,31 \\
\hline Sangramento, n (\%) & $1(0,4)$ & 0 & $1(4,3)$ & 0,10 \\
\hline
\end{tabular}

O tamanho médio dos hematomas e da equimose foi de $2,3 \pm 1,0 \mathrm{~cm}$ e $2,3 \pm 0,5 \mathrm{~cm}$, respectivamente.

Foram relatados outros desconfortos percebidos pelos pacientes, além dos questionados pelas pesquisadoras. Os mais citados pelos pacientes foram dispneia $(2,6 \%)$, cefaleia $(2,2 \%)$, tontura $(1,3 \%)$ e dor de estômago $(1,3 \%)$.

\section{DISCUSSÃO}

Neste estudo, identificaram-se os desconfortos relatados por pacientes submetidos a cateterismo cardíaco pelas vias de acesso femoral ou radial, possibilitando subsídios para melhoria da assistência prestada pela equipe de saúde. A identificação das queixas mais frequentes dos pacientes no cotidiano da prática assistencial da Hemodinâmica é necessária, pois permite estabelecer um cuidado qualificado e voltado para a resolução dos problemas.

A amostra deste estudo foi composta majoritariamente por pacientes do sexo masculino e com média de idade de 60,2 anos, o que revela que pessoas com idade mais avançada estão sendo submetidas, cada vez mais, a procedimentos percutâneos. O risco mais elevado de complicações vasculares no sítio de punção ocorre geralmente em pacientes idosos e com a utilização da via femoral. ${ }^{7}$ A identificação dos fatores de risco para complicações vasculares, durante ou após tais procedimentos, é importante para o desenvolvimento de protocolos a fim de impedi-las ou minimizá-las. ${ }^{7}$

Apesar disso, pôde-se verificar pelos achados do presente estudo que não houve diferença quanto às complicações vasculares em ambas as vias de acesso (radial e femoral). No entanto, o estudo A Randomized Comparison of Percutaneous Transluminal Coronary Angioplasty by the Radial, Brachial and Femoral Approaches: The Access Study, ou simplesmente Access, como é denominado, que comparou 900 pacientes submetidos à intervenção coronária percutânea pelas técnicas radial, braquial e femoral, encontrou significativa diminuição das complicações relacionadas à via de acesso radial, em relação às demais. ${ }^{8}$ Já em 
meta-análise que avaliou 3.224 pacientes em 12 estudos randomizados, comparando as técnicas radial e femoral, para realização de coronariografia e procedimentos de intervenção coronária percutânea, foi demonstrado que a abordagem radial é segura e efetiva quando comparada à técnica femoral, com menos complicações da via de acesso (odds ratio - OR = 0,20; intervalo de confiança de 95\% - IC 95\% 0,09-0,42; $p<0,0001)$, mesmo às custas de maiores chances de falência do procedimento $(\mathrm{OR}=3,30$; IC 95\% $1,63-6,71 ; p<0,001){ }^{9}$

Os resultados deste estudo demonstram que os pacientes que realizaram procedimentos por via de acesso femoral relataram maiores desconfortos com relação à dor lombar e ao mal-estar, com diferença entre os grupos. Essas queixas podem estar diretamente relacionadas ao tempo prolongado de repouso no leito. Estudo randomizado com 169 pacientes foi desenvolvido com o objetivo de avaliar os efeitos de colocar peso (saco de areia) no local do acesso femoral após o procedimento e mudar a posição dos pacientes no leito, em relação à taxa de complicações vasculares e à gravidade da dor lombar relacionada com a duração do repouso pós-procedimento. ${ }^{10}$ Os resultados desse estudo apontaram que a dor nas costas foi relatada com mais frequência nos pacientes cujas posições não foram alteradas e cujas as cabeceiras das camas não foram levantadas $(p<0,05) .{ }^{10} \mathrm{O}$ mesmo estudo sugeriu que, para aumentar o conforto e diminuir a dor lombar, os pacientes podem mudar sua posição no leito, assim como a cabeceira pode ser posicionada em cerca de 30 ou $45^{\circ} .{ }^{10}$ Dados de um ensaio clínico randomizado que avaliou o efeito da mobilização intermitente no leito após a realização do cateterismo por via femoral mostraram que os pacientes do grupo intervenção relataram menos dor lombar que aqueles do grupo controle $(p=.00)$. Não houve diferença significativa entre os grupos quanto à presença de sangramento. ${ }^{11}$

A mudança de posição no leito pode igualmente reduzir a dor, promovendo o conforto físico e minimizando os possíveis sentimentos negativos que os pacientes têm em relação à angiografia coronária. ${ }^{12}$ Um estudo quasi-experimental realizado com 105 pacientes para investigar o efeito de três protocolos de posicionamento sobre dor nas costas, frequência cardíaca, pressão arterial e complicações vasculares após cateterismo cardíaco concluiu que mudar de posição na cama e usar um travesseiro de apoio durante as primeiras horas após o cateterismo cardíaco pode efetivamente minimizar a dor e a instabilidade hemodinâmica, sem aumentar as complicações vasculares. ${ }^{12}$ Uma outra estratégia para redução da dor lombar é o uso de dispositivos como o Siriraj Leg Lock $^{\circledR}$, que têm por objetivo imobilizar somente o membro puncionado, permitindo a mobilidade de outras partes do corpo. Um ensaio clínico randomizado que avaliou sua utilização em 51 pacientes submetidos à intervenção coronária percutânea do grupo intervenção mostrou redução significativa da dor lombar em relação ao grupo controle $(p<0,001){ }^{13}$

Diversos estudos têm demonstrado que é possível reduzir o tempo de repouso absoluto no leito. Um estudo realizado em São Paulo comprovou que é isso é possível com a utilização de cateteres $4 \mathrm{~F}$ por via femoral, pois, mesmo no caso de intervenção coronária percutânea, a deambulação precoce (média de $60 \pm$ 5 minutos) não acarretou mais complicações hemorrágicas, além de ser mais simples, menos traumática e menos invasiva. ${ }^{14}$ Já outro estudo, que realizou um ensaio clínico randomizado, no qual os pacientes submetidos à intervenção via femoral utilizando introdutor arterial $6 \mathrm{~F}$ e com o tempo de coagulação ativada $<350$ segundos tiveram a bainha retirada ao término do procedimento e deambularam após 3 horas de repouso, revelou que a observação cuidadosa da enfermagem, após intervenção percutânea, com retirada do introdutor arterial e deambulação precoce, oferece conforto, segurança e satisfação ao paciente. ${ }^{4}$

Os achados deste estudo motivam o desenvolvimento de estratégias para minimizar as queixas mais comuns e proporcionar um atendimento mais humanizado, aliviando dor física e psíquica dos pacientes. Os cuidados de enfermagem antes e depois dos procedimentos devem ser direcionados para a prevenção e a detecção de complicações. ${ }^{15} \mathrm{~A}$ atenção ao paciente deve ser criteriosa, pois, muitas vezes, pacientes com dor podem apresentar elevação da pressão arterial e, com isso, ocasionar sangramento no local da punção e, em algumas situações, levar a complicações mais graves.

Entre outros desconfortos relatados pelos pacientes, destacamos a cefaleia, a falta de ar e a tontura. Esses dados demonstram que o paciente, no período peri-procedimento, tem diversas queixas, que podem ser prevenidas e controladas pela equipe multidisciplinar. Deve-se considerar que o período de espera para a realização do exame, as individualidades de cada paciente, as intercorrências que surgem durante os procedimentos e os resultados inesperados são estressores e agravam ainda mais o estado físico/psíquico. Frente a isso, a equipe deve estar atenta a esses sinais e sintomas para, na medida do possível, amenizá-los.

Um estudo que avaliou os fatores que predizem o nível de desconforto após angiografia coronária mostrou que os pacientes que tiveram experiência anterior com esses procedimentos receberam informações prévias sobre o próximo procedimento, não apresentaram disúria e dormiram bem após a conduta. Esse pacientes também foram menos propensos a se queixarem de desconforto. ${ }^{16}$ Por fim, torna-se importante lembrar que cada paciente tem particularidades, como patologias de coluna vertebral preexistentes (hérnia de disco e cirurgias anteriores), dificuldade urinária prévia (retenção urinária e hiperplasia de próstata), maior sensibilidade 
à dor, fraqueza, dificuldade motora e cognitiva, o que, muitas vezes, está relacionado à idade avançada e/ou a doenças associadas. Essas informações devem ser investigadas pela equipe de profissionais antes do procedimento, para que seja realizado um planejamento individualizado para minimizar os desconfortos. Como uma das estratégias para que esses problemas não sejam potencializados, a escolha da via de acesso, quando possível, pode ser útil.

\section{CONCLUSÕES}

Ao avaliarmos os desconfortos dos pacientes submetidos a cateterismo cardíaco pelas vias de acesso femoral e radial, identificou-se que o desconforto predominante pós-punção femoral foi a lombalgia e, naqueles submetidos à punção radial, foi a dor no local da punção. Ao compararmos os grupos, verificou-se que pacientes que realizaram procedimentos por via de acesso femoral relataram maiores desconfortos com relação à dor lombar e ao mal-estar geral.

Os achados deste estudo remetem a recomendações para a prática clínica que promova a qualidade da assistência, como instituir medidas de conforto ao paciente, tais como o uso de coxins, mudança de decúbito, auxílio na deambulação, ambiente acolhedor e monitoramento constante dos sinais vitais. Além disso, a identificação dos fatores de risco para complicações, no leito vascular ou no sítio da punção, cria a oportunidade para um cuidado mais individualizado. A criação de protocolos pode incluir medidas básicas e avançadas de conforto, de acordo com a via de acesso escolhida para o procedimento, tornando o período de repouso menos cansativo e incômodo. É fundamental que a equipe esteja atenta aos sinais de desconforto manifestados pelos pacientes para prontamente minimizá-los, promovendo uma recuperação mais precoce e uma assistência mais humanizada.

\section{CONFLITO DE INTERESSES}

Não há

\section{FONTE DE FINANCIAMENTO}

Não há

\section{REFERÊNCIAS}

1. Nunes GL, Oliveira AT, Alves L, Alfonso T. Influência da curva de aprendizado no sucesso e na ocorrência de complicações associadas aos procedimentos pela via radial. Rev Bras Cardiol Invasiva. 2007;15(2):115-8.
2. Armendaris MK, Azzolin KO, Alves FJMS, Ritter SG, Moraes MAP. Incidência de complicações vasculares em pacientes submetidos à angioplastia coronariana transluminal percutânea por via arterial transradial e transfemoral. Acta Paul Enferm. 2008;21(1):107-11.

3. Botelho R, Nunes GL. Especialistas contam por que preferem a técnica transradial na cinecoronariografia e na cardiologia intervencionista. J SBHCl. 2008;11(3):40-5.

4. Augustin AC. Retirada precoce do introdutor arterial com TCA acima de 180: Vantagens e desvantagens para o paciente e implicações para a enfermagem. Rev Bras Cardiol Invasiva. 2006;14(4):248-52.

5. Araújo $\mathrm{CM}$, Mangione JA. Diferenças e semelhanças dos resultados de acordo com as diferentes vias de acesso. Rev Bras Cardiol Invasiva. 2006;14(3):273-9.

6. Rossato G, Quadros AS, Sarmento-Leite R, Gottschall CAM Análise das complicações hospitalares relacionadas ao cateterismo cardíaco. Rev Bras Cardiol Invasiva 2007;15(1):44-51.

7. Dumont CJP, Keeling AW, Bourguignon C, Sarembock IJ, Turner M. Predictors of vascular, complications post diagnostic cardiac catheterization and percutaneous coronary interventions. Dimens Crit Care Nurs. 2006;25(3):137-42.

8. Kiemeneij F, Laarman GJ, Odekerken D, Slagboom T, van der Wieken R. A randomized comparison of percutaneous transluminal coronary angioplasty by the radial, brachial and femoral approaches: the access study. J Am Coll Cardiol. 1997; 29(6):1269-75.

9. Agostoni P, Biond-Zoccai GG, Benedictis ML, Rigatturi S, Turri $M$, Anselmi M, et al. Radial versus femoral approach for percutaneous coronary diagnostic and interventional procedures: systematic overview and meta-analysis of randomized trials. J Am Coll Cardiol. 2004;44(2):349-56.

10. Yilmaz E, Gürgün C, Dramali A. Minimizing short-term complications in patients who have undergone cardiac invasive procedure: a randomized controlled trial involving position change and sandbag. Anadolu Kardiyol Derg. 2007;7(4): 390-6.

11. Bakhshi F, Namjou Z, Andishmand A, Panabadi A, Bagherinasab $M$, Sarebanhassanabadi $M$. Effect of positioning on patient outcomes after coronary angiography: a single-blind randomized controlled trial. J Nurs Res. 2014;22(1):45-50.

12. Rezaei-Adaryani M, Ahmadi F, Mohamadi E, Asghari-Jafarabadi $M$. The effect of three positioning methods on patient outcomes after cardiac catheterization. J Adv Nurs. 2009;65(2): 417-24.

13. Pornratanarangsi S, Boonlert S, Duangprateep A, Wiratpintu $\mathrm{P}$, Waree W, Tresukosol D, et al. The effectiveness of "Siriraj Leg Lock" brace on back pain after percutaneous coronary intervention: PCI. Med Assoc Thai. 2010;93 Suppl 1:S35-42.

14. Teixeirense PT, Gubolino LA, Bragalha AMLA, Toledo JFB, Franceschine J, Colombo $\mathrm{O}$, et al. Análise temporal dos resultados imediatos com a aplicação da punção transradial na intervenção coronária percutânea. Rev Bras Cardiol Invasiva. 2006;14(4):380-5.

15. Bowden T. Evidence-based care for patients undergoing coronary angiography. Br J Nurs. 2009;18(13):776-83.

16. Park AR, Choi JY. Factors predicting patient discomfort after coronary angiography. J Korean Acad Nurs. 2009;39(6):860-7. 